investigated, the vitamin is found in the plasma and other tissues. Because it is always present it is assumed to be essential, and because it is an oxidation reduction catalyst it is described as "an indispensable hydrogen carrier in the metabolism of animals". It is surely not wise to disguise our ignorance about a fundamental metabolic problem by making sweeping and unsupported statements. Our knowledge is likely to be increased only by an accumulation of precisely the kind of facts given in Kalnenas's own letter.

A. L. BaCharach

Glaxo Laboratories, Ltd.,

Greenford, Middlesex.

${ }^{1}$ Nature, 169, 836 (1952).

"Harris, L. J., "Vitamins : A Digest of Current Knowledge" (Churchill, Itc., London, 1951).

${ }^{3}$ Damron, C. M.., Monier, M. M., and Roe, J. H., J. Biol. Chem., 195, $599(1952)$.

\section{A Simple Rotating-Cell Double-Beam Unit for Spectrometers}

SINCE publishing our preliminary report on this unit $^{1}$, we have been able to carry out further tests upon materials having strong absorption bands in the region of atmospheric water vapour absorption. From these experiments, we have now found that our system is not a true double-beam system, since it measures the difference between the energies of the two beams, and not their ratio. Consequently the absorption due to atmospheric water vapour and carbon dioxide is not completely removed, but appears as a function of the absorption of the test material at these wave-lengths.

Polystyrene sheet was chosen as our first test material for ease of manipulation. This choice was particularly unfortunate, since the wave-lengths of the strong absorption bands of this material do not fall near those of strong water-vapour absorption. It is for this reason, together with the low resolution used in our preliminary experiments, that the effects of atmospheric water-vapour absorption are not apparent in our published spectrum.

Brown, Holliday and Trotter ${ }^{2}$ have pointed out to us that our rotating-cell system is really an 'inverted' single-beam system with a variable 100 per cent absorption line. They have also brought to our notice that an analogous principle was suggested some years ago by Hilger and Watts, Ltd., for the alignment of the entrance and exit slits of their D.209 doublebeam spectrometer. In this method, the two thermocouple detectors are connected in opposition. If the slits are correctly aligned, no signal is observed when passing through a region of atmospheric watervapour absorption. When a sample is introduced into one beam, however, the record gives the absorption spectrum of the sample minus the water-vapour absorption : the stronger the absorption band of the sample, the greater the amount of water-vapour absorption subtracted.

One advantage of our system is that it can be used for the investigation of weak sample bands in the region of atmospheric water-vapour absorption when it is not possible to eliminate the water absorption beyond a few per cent of the total energy. Residual water absorption of this magnitude would be negligible for weak sample absorption and, consequently, weak bands could be identified with certainty. With this in view, a method similar in principle to, but simpler than our rotating cell, can be used for the examination of solid films'. In this method, portions of the specimen are attached to the circumference of a disk mounted in place of the conventional chopping sector.

Our method also enables the strong aliphatic CHband due to the 'Nujol' used in mulls to be reduced considerably by using a 'Nujol' film in the blank cell. This reduction in intensity of aliphatic $\mathrm{CH}$-bands enables the aromatic CH-bands of substances under investigation to be more clearly identified.

We would like to apologize to any workers who may have started to construct a rotating-cell system on the basis of our earlier communication. In these circumstances, it is not proposed to publish further details of the mechanism used for rotating the cells.

J. D. S. GOULDEN

S. S. RANDALL

Research Department,

Biochemistry Division,

Boots Pure Drug Co., Ltd., Nottingham. May 26.

${ }^{1}$ Goulden, J. D. S., and Randall, S. S., Nature, 169, 748 (1952).

${ }^{2}$ Brown, L., Holliday, P., and Trotter, I. F. (private communication).

\section{Production of Electric Charges on Water Drops}

A RECENT communication ${ }^{1}$ by Gill and Alfrey implies that we have interpreted the potential differences accompanying the freezing of dilute aqueous solutions as contact potentials. On the contrary, we agree with Gill and Alfrey that "a contact potential difference of 230 volts seems most unlikely". We further believe that the implied interpretation is completely untenable, because the observed potential differences cause the flow of currents of the order of 1 ramp. The statement in question is that "the potential barrier is at the waterice interface". This statement was intended to convey that the charge separation occurs at the interface, and that each ion involved in the charge separation must cross a potential-barrier equal in magnitude and sign to the measured potential difference between the ice and water.

Gill and Alfrey suggest that certain impurities in the water might reverse the sign of the potential differences as distilled water is frozen. We believe that our experiments ${ }^{2}$ show clearly that the sign and magnitude of the potential difference developed and the quantity of charge separated during freezing are functions of the kind and amount of contaminants in solution with the water. It was only after recognition of these facts that we were able to escape the irreproducibility that apparently plagued Gill and Alfrey in their experiments, and our conclusion that the potential differences result from the selective incorporation of foreign ions into the growing ice structure is based in part on these facts.

It is suggested by Gill and Alfrey that the potential differences arise because the surface charges which cause the contact potential difference between ice and water are continuously frozen into the ice. They state that "the reverse process does not appear to take place when melting occurs, for the much higher conductivity of water would prohibit the building up of a volume distribution of charge in the water". It should be pointed out that, in an experiment such as they describe, both ice and water are present whether freezing or melting is going on. The con- 